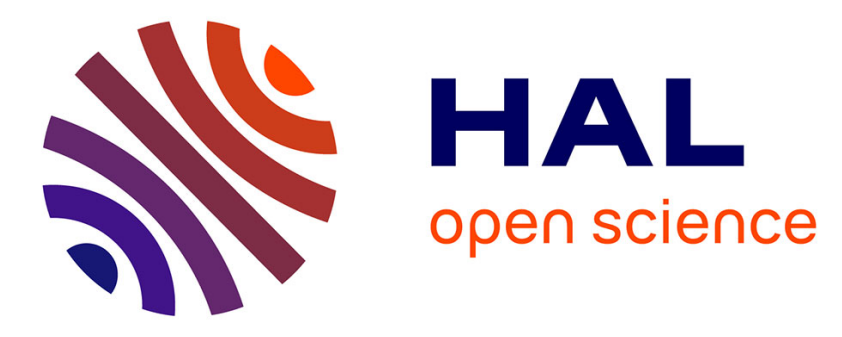

\title{
Valorisation des sédiments du barrage de Zardezas (Algérie): Caractérisation et aptitude au compactage des sédiments
}

Selma Bellara, Daniel Levacher, Salim Mezazigh, Mustapha Hidjeb

\section{- To cite this version:}

Selma Bellara, Daniel Levacher, Salim Mezazigh, Mustapha Hidjeb. Valorisation des sédiments du barrage de Zardezas (Algérie): Caractérisation et aptitude au compactage des sédiments. XVIème Journées Nationales Génie Côtier - Génie Civil XVIème, Dec 2020, Le Havre, France. pp.571-580, 10.5150/jngcgc.2020.064 . hal-03355189

HAL Id: hal-03355189

https://hal-normandie-univ.archives-ouvertes.fr/hal-03355189

Submitted on 27 Sep 2021

HAL is a multi-disciplinary open access archive for the deposit and dissemination of scientific research documents, whether they are published or not. The documents may come from teaching and research institutions in France or abroad, or from public or private research centers.
L'archive ouverte pluridisciplinaire HAL, est destinée au dépôt et à la diffusion de documents scientifiques de niveau recherche, publiés ou non, émanant des établissements d'enseignement et de recherche français ou étrangers, des laboratoires publics ou privés. 


\title{
Valorisation des sédiments du barrage de Zardezas (Algérie): Caractérisation et aptitude au compactage des sédiments
}

\author{
Selma BELLARA ${ }^{1,2}$, Daniel LEVACHER ${ }^{1}$, Salim MEZAZIGH ${ }^{1}$, \\ Mustapha HIDJEB ${ }^{2}$
}

1. Normandie Université, Unicaen, CNRS UMR 6143 M2C, 14000, Caen, France. daniel.levacher@unicaen.fr; salim.mezazigh@unicaen.fr;

2. Université 20 Août 1955, Laboratoire LMGHU, Skikda, Algérie.

blrselma@hotmail.com

\section{Résumé:}

En raison du taux élevé d'envasement des barrages et autres retenues d'eau, zones de sédimentations en rivières (oueds) ou à l'embouchure de celle-ci, la gestion durable des sédiments est devenue une véritable préoccupation nationale en Algérie. Cependant, une gestion durable des sédiments dragués dans ces zones implique une recherche de filières de valorisation à proximité des lieux de prélèvements. Une valorisation en matériau routier, de remblai, voire de remplissage peut constituer une solution potentiellement acceptable mais aussi, qui répond aux besoins d'aménagement du territoire Algérien. Une approche méthodologique de valorisation consiste à caractériser le sédiment et à définir ses propriétés d'aptitude au compactage pour répondre à la mise en place de ces matériaux routiers. Cette approche propose pour des sédiments dragués en-amont du barrage de Zardezas (Skikda, Algérie) pour un réemploi en tant que matériaux de remplacement en techniques routières. La caractérisation minimale de ces sédiments et leurs caractéristiques, sont établies et discutées. L'aptitude au compactage a été effectuée selon deux essais : l'essai Proctor normal et l'essai de compactage miniature à énergie équivalente. Les résultats d'essai de compactage sont analyses en relation avec les caractéristiques du sédiment et les moyens d'essai mis en œuvre. Les conclusions portent sur la caractérisation minimale et la potentialité de valorisation en matériau routier ou de remblai et sur les avantages de l'essai de compactage miniature utilisé.

\section{Mots-clés:}

Sédiment de barrage, Caractérisation, Valorisation, Aptitude au compactage, Traitement aux liants, Proctor miniature, Gestion des sédiments.

\section{Introduction}

Le ministère algérien des Ressources en eau et de l'environnement a indiqué en septembre 2018 que le secteur recense 80 barrages en cours d'exploitation et 5 grands barrages en cours de réalisation, ces derniers seront progressivement réceptionnés en 2020. Cependant, certains de ces barrages, 114 identifiés en 2008 (MEKERTA et al., 2008) ne sont plus exploités et environ 20 grands barrages sont menacés par le 


\section{Thème 6 - Gestion durable des zones littorales et estuariennes}

comblement à court terme (REMINI \& HALLOUCHE, 2007). A titre d'exemple, le barrage de Zardezas a atteint une vitesse d'envasement de 0.4 million de $\mathrm{m}^{3} /$ an entre 2003 et 2006 (TOUMI \& REMINI, 2020), d'où la nécessité de dragage. Néanmoins, les opérations de dragage ont généré et génèrent d'importants volumes de sédiments à stocker puis à valoriser tout en respectant la législation environnementale en vigueur. Le principal domaine ciblé est le génie routier, en particulier les sous-couches de structures routières pour lesquelles un grand volume de sédiments pourrait être réutilisé. Dans le cadre du développement durable et d'économie des ressources, il est recommandé que la quantité de liants hydrauliques (ciment, chaux) et d'apports granulaires soit réduite. Cette étude aborde la possibilité de réutilisation des sédiments dragués du barrage de Zardezas (Algérie) et de leur valorisation dans les sous-couches de structures routières. Les analyses physico-chimiques et minéralogiques des sédiments complétées par des essais mécaniques (compactage), permettent d'envisager leur utilisation comme matériau routier et/ou de remplissage après les avoir traités. Les résultats sont discutés afin de sélectionner le traitement à appliquer aux sédiments tout en respectant la réglementation en vigueur, sachant qu'un traitement mixte à base de chaux, de ciment avec ajout d'un matériau granulaire semble nécessaire, comme le montrent des études récentes (BANOUNE et al., 2016 ; BENAISSA et al., 2016).

\section{Identification des sédiments à valoriser}

\subsection{Origine et lieu de prélèvement des sédiments}

L'étude porte sur les sédiments du barrage de Zardezas situé au nord-est de l'Algérie. Les sédiments ont été prélevés en surface en amont du barrage à l'aide d'une pelle (figure 1). Ils ont été transportés au laboratoire dans des sacs hermétiques, séchés $\left(<50^{\circ} \mathrm{C}\right)$ pour éviter toute modification de leurs composantes chimiques notamment organiques, désagrégés puis homogénéisés pour effectuer les essais de caractérisation.

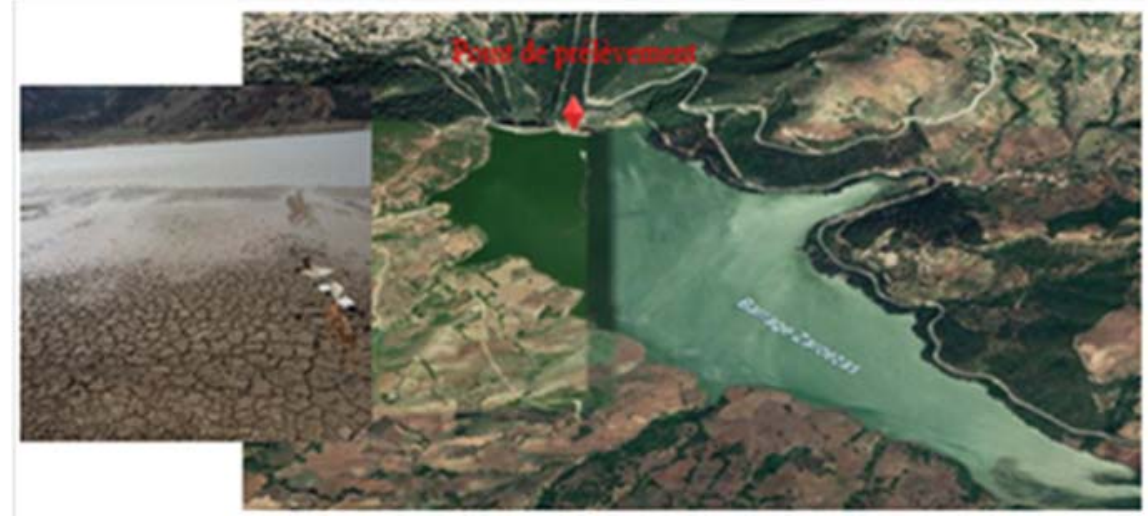

Figure 1. Lieu de prélèvement des sédiments. 


\section{XVIèmes Journées Nationales Génie Côtier - Génie Civil \\ Le Havre 2020}

\subsection{Caractérisation physique des sédiments}

\subsubsection{Analyse granulométrique}

Deux méthodes pour la détermination de la distribution granulométrique en taille de particules des sédiments, ont été utilisées. La première selon les normes françaises NF P94-041 et 057 est relative au tamisage par voie humide pour les éléments de diamètre supérieur à $80 \mu \mathrm{m}$ et à la sédimentométrie pour les éléments inférieurs à $80 \mu \mathrm{m}$. La deuxième méthode s'appuie sur une technique plus précise, il s'agit d'une analyse granulométrique par diffraction laser (LS13 320 de Beckman Coulter). Elle s'applique à des sols fins dont la taille maximale ( $D_{\text {max. }}$ ) n'excède pas $4 \mathrm{~mm}$. Les résultats obtenus sont présentés sur la figure 2 et dans le tableau 1. Deux échantillons ont été considérés pour chaque méthode. Les granulométries tout au moins pour la partie grossière semblent s'accorder compte-tenu de la variabilité naturelle des sédiments. Ce sédiment est un sol fin $\left(\mathrm{D}_{\max .}<2 \mathrm{~mm}\right)$.

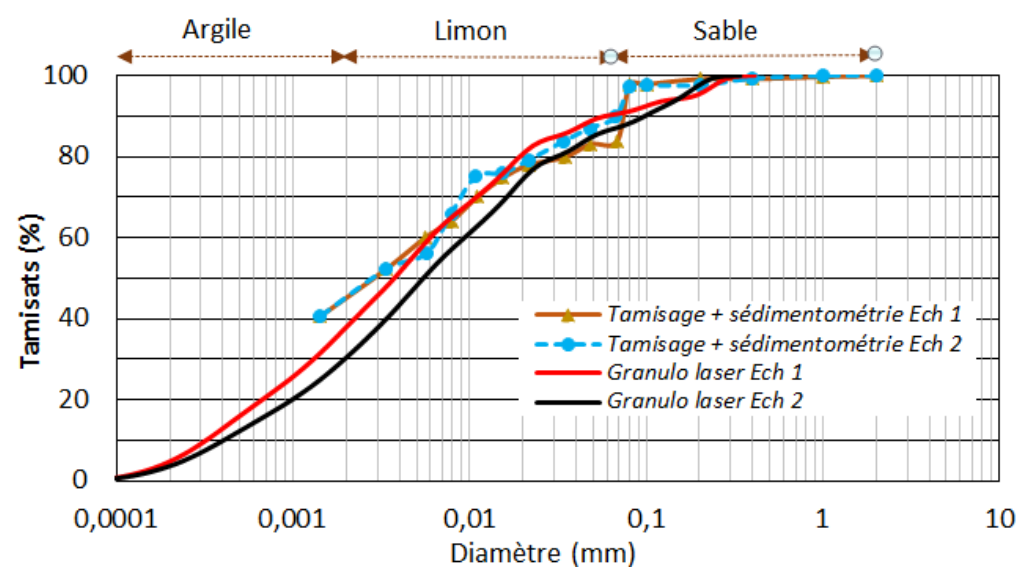

Figure 2. Distributions granulométriques du sédiment à valoriser.

Le tableau 1 situe la nature granulométrique du sédiment pour chaque méthode. Les résultats montrent une prédominance de la fraction fine (argiles et limons). La fraction sableuse ne dépasse pas $17 \%$.

Tableau 1. Nature granulométrique du sédiment à valoriser.

\begin{tabular}{llll}
\hline \multirow{2}{*}{ Echantillon } & $\begin{array}{l}\text { Argile (\%) } \\
\boldsymbol{D}<\mathbf{2 \mu \boldsymbol { m }}\end{array}$ & $\begin{array}{l}\text { Limon (\%) } \\
\mathbf{2} \boldsymbol{\mu m}<\boldsymbol{D}<\mathbf{6 3} \boldsymbol{\mu m}\end{array}$ & $\begin{array}{l}\text { Sable (\%) } \\
\mathbf{6} \boldsymbol{\mu m}<\boldsymbol{D}<\mathbf{2 m m}\end{array}$ \\
\hline Tamisage +Sédim. Ech1 & 45,0 & 38,0 & 17,0 \\
Tamisage +Sédim. Ech2 & 45,5 & 43,0 & 11,5 \\
Valeur moyenne & 45,3 & 40,5 & 14,3 \\
\hline Granulolaser Ech1 & 38,0 & 52,0 & 10,0 \\
Granulolaser Ech2 & 30,5 & 56,0 & 13,5 \\
Valeur moyenne & 34,3 & 54,0 & 11,8 \\
\hline
\end{tabular}




\section{Thème 6 - Gestion durable des zones littorales et estuariennes}

Les coefficients de courbure $\mathrm{C}_{\mathrm{c}}$ et d'uniformité $\mathrm{C}_{\mathrm{u}}$ ont été également calculés (tableau 2). On constate avec un $\mathrm{C}_{\mathrm{u}}$ largement supérieur à 2 que la répartition granulométrique est très étalée. Le coefficient de courbure $\mathrm{C}_{\mathrm{C}}$ inférieur à 1 , signifie que la granulométrie est mal graduée, la continuité est mal répartie.

\subsubsection{Plasticité, argilosité, matière organique et masse volumique du sédiment}

D'autres paramètres géotechniques sont nécessaires à l'identification, à la classification et au choix de la filière de valorisation des sédiments. Les limites d'Atterberg ( $W_{L}, W_{P}$ et $\left.I_{p}\right)$ et la valeur de bleu de méthylène VBS ont été déterminées respectivement selon les normes NF P94-052 et NF P94-068. La valeur du pH a été mesurée à l'aide d'un pHmètre en suivant la norme NF ISO 10390. La masse volumique des grains solides (NF P 18558 ) et la teneur en matière organique (MO) par perte au feu complètent ces données. Les essais ont été réalisés sur un minimum de 2 échantillons, les valeurs moyennes sont récapitulées au tableau 2.

Tableau 2. Paramètres de caractérisation des sédiments à valoriser.

\begin{tabular}{|c|c|c|c|}
\hline Paramètres & Valeur moyenne & Paramètres & Valeur moyenne \\
\hline Granulométrie par tamisage & & $\rho_{s}\left(\mathrm{~g} / \mathrm{cm}^{3}\right)$ & 2,66 \\
\hline Argile (\%) $D \leq 2 \mu m$ & 45,25 & pH & 8,35 \\
\hline Limon (\%) $2 \mu \mathrm{m}<D<63 \mu \mathrm{m}$ & 40,5 & $W_{L}(\%)$ & 52,1 \\
\hline Sable (\%) $63 \mu \mathrm{m}<D<2 \mathrm{~mm}$ & 14,25 & $W_{P}(\%)$ & 22,2 \\
\hline Granulolaser & & $I_{P}(\%)$ & 29,9 \\
\hline Argile (\%) $D \leq 2 \mu m$ & 34,25 & VBS (\%) & 4,41 \\
\hline Limon (\%) $2 \mu m<D<63 \mu m$ & 54 & MO (\%) & 6,29 \\
\hline Sable (\%) $63 \mu \mathrm{m}<D<2 \mathrm{~mm}$ & 11,75 & $\mathrm{CaCo}_{3}(\%)$ & 15,55 \\
\hline Cu et Cc & 21,04 et 0,96 & Activité des argiles & 0,87 \\
\hline
\end{tabular}

\subsection{Analyse minéralogique}

Le tableau 3 et la figure 3 présentent les principaux minéraux qui constituent les sédiments de Zardezas. Les résultats montrent que ces derniers se composent principalement de quartz $\left(\mathrm{SiO}_{2}\right)$, de kaolinite, d'illite, de carbonates (calcite $\mathrm{CaCo}_{3}$ et dolomite). Des teneurs faibles en chlorite et des minéraux ferrugineux sont observées.

Tableau 3. Principaux minéraux contenus dans les sédiments.

\begin{tabular}{|l|l|l|l|l|l|l|l|l|}
\hline Minéraux & Quartz & Calcite & Dolomite & Albite & Illite & Kaolinite & Chlorite & Minéraux ferrugineux \\
\hline (\%) & 33 & 11 & 3 & traces & 14 & 30 & 4 & 5 \\
\hline
\end{tabular}




\section{XVİ̀mes Journées Nationales Génie Côtier - Génie Civil \\ Le Havre 2020}

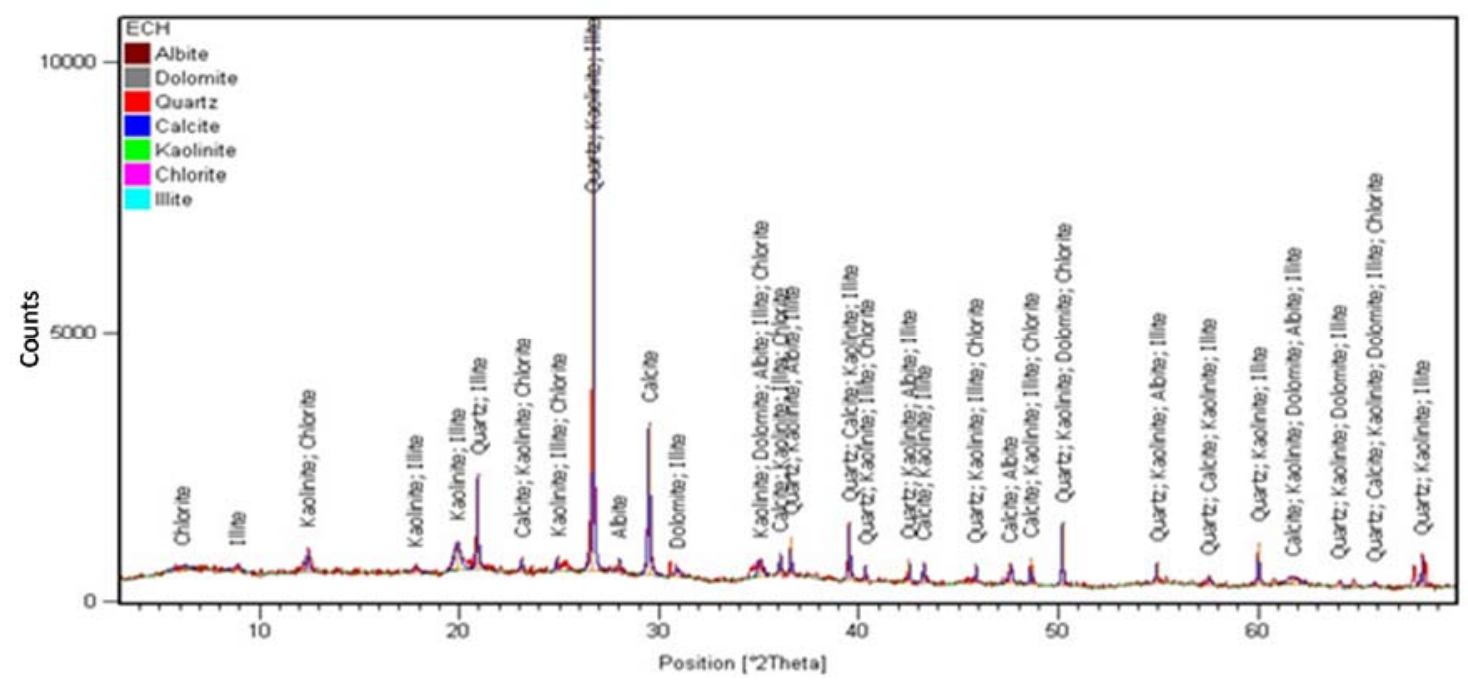

Figure 3. Diffractogramme DRX des sédiments à valoriser.

\subsection{Identification}

L'identification des sédiments se fait à l'aide de classification spécifique selon la voie de valorisation envisagée. Pour une valorisation matériau, elle s'appuie sur la classification géotechnique USCS des sols et plus spécifiquement sur la classification GTR (2000) ou GTS (2000) pour un matériau routier. Avec une teneur en MO entre 3 et 10\%, les sédiments de Zardezas appartiennent à la classe $\mathrm{F}$ et sous classe F11 et sont donc considérés comme un matériau faiblement organique selon le guide technique GTR. En se référant à la distribution granulométrique, les sédiments font partie de la classe $\mathrm{A}_{3}$ selon la classification GTR (2000), avec un diamètre inférieur ou égal à $50 \mathrm{~mm}$ et une importante fraction fine $(<80 \mu \mathrm{m})$ supérieure à $35 \%$, voir figure 4 . Cette classification est aussi celle de sédiments de barrages algériens : barrage de Fergoug (BENAISSA et al., 2016) et barrage de Merdja Sidi Abed (SEKLAOUI, 2016). Pour ce dernier barrage, la fraction fine des sédiments observée (39,1\% d'argiles et 59\% de limons) est proche de celle des sédiments étudiés.
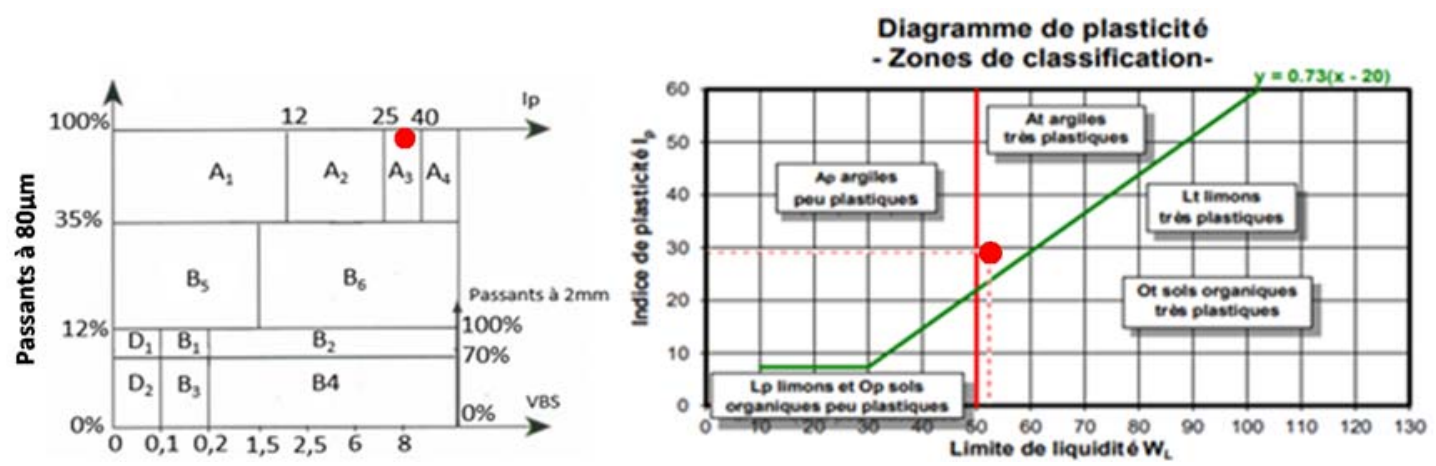

Figure 4. Classification GTR et plasticité des sédiments à valoriser. 


\section{Thème 6 - Gestion durable des zones littorales et estuariennes}

Ainsi, pour une éventuelle utilisation en couche de forme, cette catégorie de sol i.e. sédiment, implique l'utilisation d'un traitement à la chaux seule ou un traitement mixte avec des liants hydrauliques selon le GTR (2000). Selon l'abaque de Casagrande (figure 4 à droite), les sédiments de Zardezas se situent tout juste au-dessus de la ligne A appartenant au groupe des argiles inorganiques de plasticité élevée.

\section{Etude du compactage sur des volumes réduits de sédiments}

Le principe de l'essai Proctor consiste en l'application d'une énergie mécanique définie conformément aux normes sur un échantillon de sol humidifié à plusieurs teneurs en eau, afin de déterminer sa masse volumique sèche maximale $\rho_{\text {dmax }}$ pour une teneur en eau optimale WOPN. Un format miniature de l'appareil Proctor classique a été proposé dans plusieurs études antérieures (KOUASSI et al., 2000; FERBER et al., 2009; LEVACHER et al., 2017). Un Proctor miniature a été utilisé dans la présente étude, ces avantages sont la rapidité de l'opération et la faible quantité de sol utilisée qui ne dépasse pas $1 \mathrm{~kg}$ par essai. Ceci assure donc un gain de temps et de matériaux et permet d'obtenir les caractéristiques recherchées en peu de temps. Il est aisé de répéter l'essai de manière à mieux définir la courbe de compactage. Le dispositif Proctor miniature se compose de 3 parties amovibles: l'embase, le moule et la partie supérieure qui fixe le moule à l'embase. En plus, une tige support avec une masse tombante fixée sur une mini dame cylindrique en pied de la tige. Une vis de blocage permet de régler la hauteur de chute de la masse, (figure 6A). L'énergie de référence du Proctor miniature correspond à celle de l'essai Proctor normal estimée à $587.86 \mathrm{kN} . \mathrm{m} / \mathrm{m}^{3}$ (énergie par unité de volume, EpN / V). Pour rappel l'énergie délivrée est donnée par l'équation 1 :

$E_{P N}=M \cdot g \cdot H \cdot N \cdot n$

avec les notations et caractéristiques suivantes : $\mathrm{M}$, masse de la dame (2490 g); g, pesanteur $\left(9,81 \mathrm{~m} / \mathrm{s}^{2}\right) ; \mathrm{H}$, hauteur de chute $(305 \mathrm{~mm}) ; \mathrm{N}$, nombre de coups par couche ( 25 coups); $\mathrm{n}$, nombre de couches ( 3 couches) et $\mathrm{V}$, volume du moule $\left(946,69 \mathrm{~cm}^{3}\right)$.
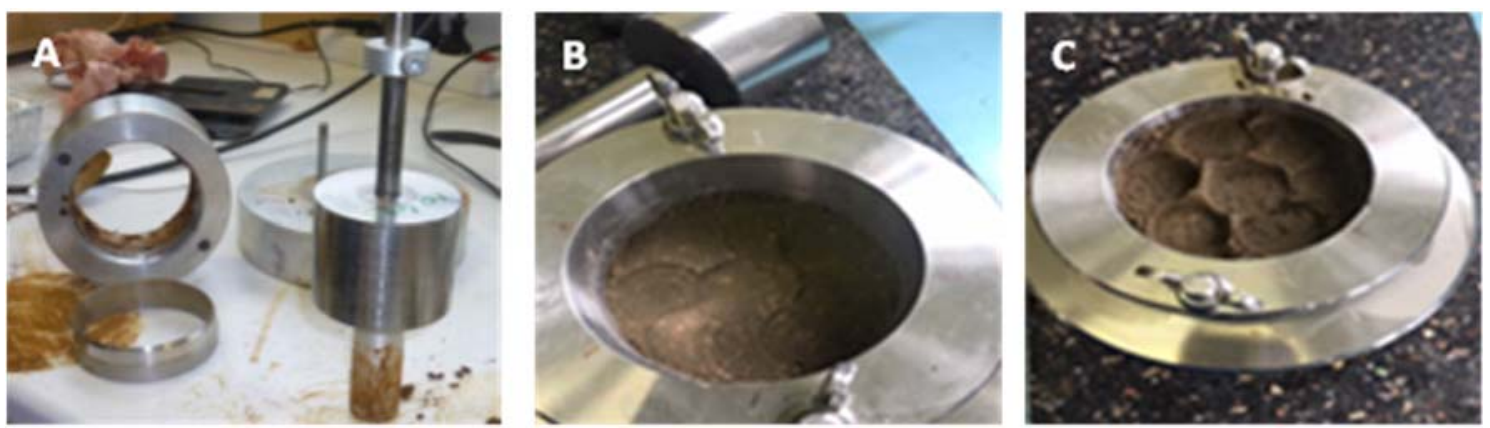

Figure 6. Proctor miniature : Appareillage et procédures avec un moule odométrique.

Quant à la procédure relative au Proctor miniature, elle consiste à placer l'échantillon de sol à l'intérieur du moule en deux couches (figures 6B et C), à compacter à l'aide d'une 


\section{XVIèmes Journées Nationales Génie Côtier - Génie Civil \\ Le Havre 2020}

dame cylindrique et appliquer sur la surface de l'échantillon 6 séries de 7 coups par couche. L'énergie Proctor miniature équivalente est de $\mathrm{E}_{b}=42.97$ N.m avec un volume de moule œdométrique égal à $\mathrm{V}_{\mathrm{b}}=73.12 \mathrm{~cm}^{3}$ et les caractéristiques de compactage inhérentes sont les suivantes : hauteur de chute de $50 \mathrm{~mm}$, une masse tombante de 1.043 $\mathrm{kg}$, un nombre de coups par couche de 42 et un nombre de couches de 2 .

\section{Essais de compactage à l'aide du Proctor miniature}

\subsection{Essais de compactage sur le sédiment brut à valoriser}

D'une part, un essai de compactage normal selon la norme NF P94-093 a été effectué sur les sédiments bruts en laboratoire. L'échantillon a été humidifié et conservé pendant $24 \mathrm{~h}$ dans des sacs hermétiques pour permettre l'infiltration d'eau entre les grains et une homogénéisation correcte de l'échantillon. D'autre part, un essai Proctor miniature a été réalisé sur les mêmes sédiments. Les échantillons de sédiments ont été humidifiés à plusieurs teneurs en eau et conservés dans des sacs pour une durée de $24 \mathrm{~h}$. Le sédiment est ensuite placé dans un moule œdométrique en 2 couches, et compacté selon la procédure détaillée précédemment, (figure 6). Pour chaque appareillage, les caractéristiques optimales sont obtenues à la fin des essais. Les courbes de compactage ainsi obtenues avec les 2 dispositifs Proctor normal (PN) et Proctor miniature (PM), montrent une légère différence de comportement au compactage. Cette différence est due peut-être à plusieurs facteurs : effet d'échelle, composition variable des sédiments, différents points de prélèvement, ... (voir figure 7, à gauche).
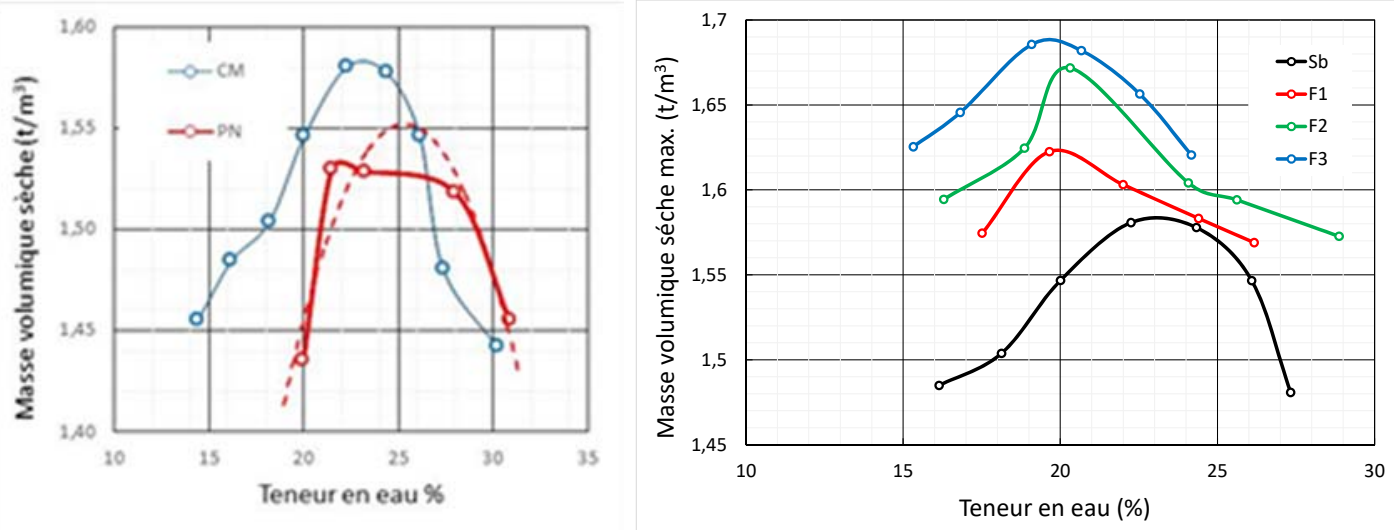

Figure 7. Courbes de compactage des sédiments bruts (gauche) et traités (droite).

\subsection{Essais de compactage sur le sédiment traité pour une valorisation routière}

Un traitement mixte classique à la chaux et au ciment avec une correction du squelette solide a été appliqué aux sédiments à valoriser selon le protocole suivant (figure 8). Les sédiments ont été d'abord humidifiés et conservés dans un sac hermétique pendant $24 \mathrm{~h}$. Le lendemain, les sédiments sont placés dans la cuve du malaxeur pour être mélangés au 


\section{Thème 6 - Gestion durable des zones littorales et estuariennes}

sable, puis à de la chaux. Le mélange est conservé pour une durée de 60 min dans un sac hermétique. Le ciment est ensuite ajouté et mélangé de nouveau pour obtenir des sédiments traités prêts à l'opération du compactage.

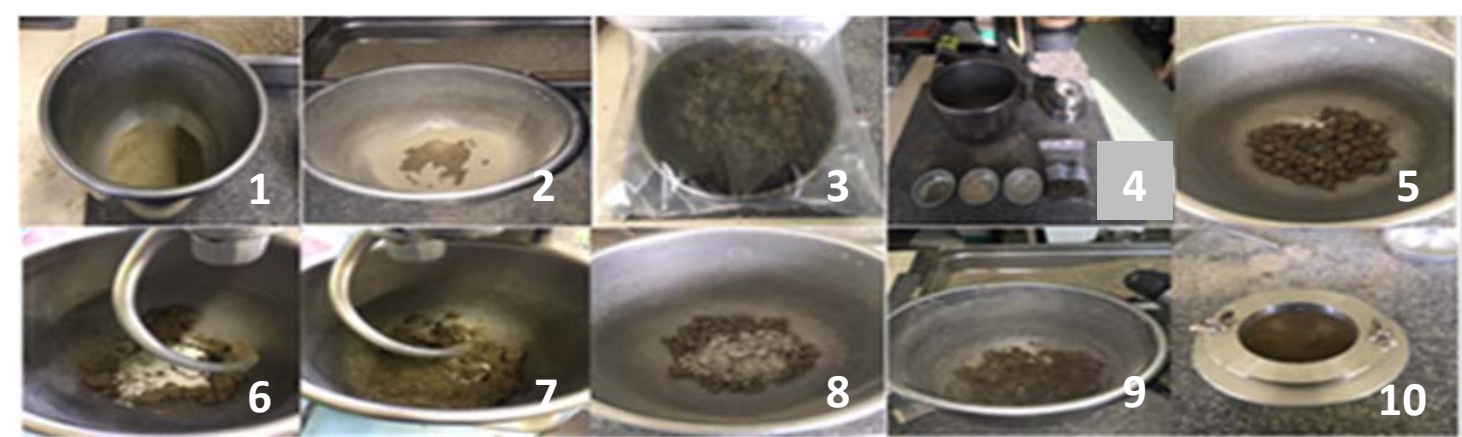

Figure 8. Etapes de préparation du sédiment traité pour l'essai de compactage.

Les essais de compactage effectués après 2 jours de préparation, ont concerné des mélanges avec un pourcentage constant de ciment et de chaux de $4 \%$ et $2 \%$ respectivement mais avec un ajout de sable de carrière variant de $3 \%(\mathrm{~F} 1), 5 \%(\mathrm{~F} 2)$ et $10 \%$ (F3). Les courbes de compactage obtenues pour les sédiments traités et à titre de comparaison celle des sédiments bruts $(\mathrm{Sb})$, sont montées à la figure 7 à droite.

\section{Analyse et discussion}

D'abord, on constate que la distribution granulométrique obtenue par les 2 méthodes montre un écart dans les résultats, ceci peut être expliqué par la différence dans le principe des méthodes utilisées, la variabilité naturelle des sédiments et également par la présence non négligeable de la matière organique $(6,29 \%)$ qui ralentit la vitesse de décantation des particules dans le cas de la sédimentométrie (ZRI, 2010). Par ailleurs, avec un coefficient $\mathrm{Cc}<1(\mathrm{Cc}=0,96)$, on peut considérer que les sédiments contiennent en grande partie une fraction fine ce qui a été confirmé par la présence dans les sédiments d'un taux de 34,3\% d'argile et de $54 \%$ de limons. Le pourcentage élevé de l'Ip et une valeur VBS $>0.02$ confirme la sensibilité des sédiments à l'eau (GTR, 2000). Ces sédiments de barrage, appartiennent à la sous classe $A_{3}$. Cette catégorie ne permet pas l'utilisation directe des sédiments pour des couches de forme. Un traitement à la chaux seule ou mixte à la chauxciment est indispensable pour cette catégorie de sol. Dans l'objectif d'évaluer l'aptitude au compactage des sédiments et l'obtention des paramètres optimaux $\left(\mathrm{W}_{\mathrm{opt}}, \rho_{\mathrm{dmax}}\right)$, des séries d'essai Proctor ont été réalisées sur les sédiments bruts et traités à la chaux et au ciment avec l'ajout d'un correcteur granulométrique. Les résultats obtenus par les 2 appareillages pour les sédiments bruts sont présentés dans le tableau 5. Ces derniers montrent une teneur en eau optimale $\left(\mathrm{W}_{\mathrm{opt}}\right)$ et une masse volumique sèche $\rho_{\mathrm{d}}$ très proches ce qui valide l'utilisation du Proctor miniature. Pour les sédiments traités à la chaux, au ciment avec un ajout de sable de carrière, on note une modification des caractéristiques 


\section{XVI'mes Journées Nationales Génie Côtier - Génie Civil \\ Le Havre 2020}

de compactage. Les courbes relatives aux mélanges de formulation $4 \%$ de ciment (C), $2 \%$ de chaux $(\mathrm{Cx})$ avec $3 \%, 5 \%$ et $10 \%$ de sable de carrière $(\mathrm{Sc})$ s'inscrivent au-dessus et à gauche de la courbe Proctor (coté sec) des sédiments bruts. Une diminution en teneur en eau optimale $\mathrm{W}_{\text {opt }}$ accompagnée d'une augmentation de la valeur de la masse volumique sèche $\rho_{\mathrm{d}}$, est clairement observée. Ceci est dû à l'influence des liants d'une part et de l'ajout du correcteur granulométrique d'autre part. Ces résultats s'accordent avec ceux d'auteurs dans la littérature. Par ailleurs, l'augmentation en pourcentage de sable de carrière peut, cependant, avoir un effet sur la perméabilité du mélange et également sur la résistance à la compression. Ces effets sont à confirmer par des essais complémentaires.

Tableau 5: Paramètres optimaux de compactage.

\begin{tabular}{|c|c|c|c|c|c|}
\hline Sédiments bruts & $\rho_{d}\left(M g / m^{3}\right)$ & Wopt (\%) & Sédiments traités & $\rho_{d}\left(M g / m^{3}\right)$ & Wopt (\%) \\
\hline Proctor normal standard & 1,55 & 25,20 & $F 1: C 4 \%+C x 2 \%+S c 3 \%$ & 1,62 & 20,0 \\
\hline \multirow[t]{2}{*}{ Proctor normal miniature } & 1,58 & 23,00 & $F 2: C 4 \%+C x 2 \%+S c 5 \%$ & 1,67 & 20,3 \\
\hline & & & F3: $C 4 \%+C x 2 \%+$ Sc $10 \%$ & 1.69 & 19.7 \\
\hline
\end{tabular}

\section{Conclusions}

Cette étude a permis de mener une caractérisation physico-chimique, minéralogique et une connaissance de l'aptitude au compactage des sédiments du barrage de Zardezas (Algérie). La teneur en constituants a montré une prédominance de la fraction fine avec un pourcentage de $88.25 \%$. Les sédiments sont des argiles limoneuses très plastiques comme l'atteste les valeurs élevées des limites d'Atterberg. Aussi, une teneur en matière organique supérieure à 3 mais inférieure à 10 est obtenue. Cette présence de matière organique pourrait perturber le déroulement de l'hydratation, la prise hydraulique ou pouzzolanique des mélanges (GTS, 2000) même si ces sédiments sont en sous classe F11 correspondant à un matériau faiblement organique. Par ailleurs, en se référant à la distribution granulométrique, les sédiments font partie de la classe $A_{3}$. Les sédiments ne sont pas aptes en leur état naturel à être utilisés en matériau routier. La sensibilité à l'eau et la plasticité élevée des sédiments impliquent un traitement à la chaux seule ou un traitement mixte avec des liants hydrauliques pour une éventuelle utilisation en couche de forme (GTR, 2000). L'étude du comportement au compactage des sédiments bruts a été menée à l'aide d'un Proctor normal et d'un Proctor miniature. Ces essais ont permis de déterminer la teneur en eau optimale et la masse volumique sèche avec un assez bon accord entre les résultats pour les 2 appareillages. L'utilisation du Proctor miniature a permis de gagner du temps et de réduire la quantité de sédiments nécessaire à l'étude des différents mélanges avec de la chaux, du ciment et un sable correcteur. Cette étude nécessite d'être complétée par d'autres essais sur les mélanges : essais mécaniques, essais de durabilité et essais environnementaux, afin de valider une formulation qui pourra être retenue pour une éventuelle utilisation en couche de forme en technique routière. 


\section{Thème 6 - Gestion durable des zones littorales et estuariennes}

\section{Références}

BANOUNE B., MELBOUCI B., ROSQUOËT F., LANGLET T. (2016). Treatment of river sediments by hydraulic binders for valorization in road construction, Bull. Eng. Geol. Environ, Vol. 75, pp 1505-1517. https://doi.org/10.1007/s10064-015-0844-4

BENAISSA A., ALOUI Z., GHEMBAZA, M. S., LEVACHER D., SEBAIBI Y. (2016). Behavior of sediment from the dam Fergoug in road construction, Advances in concrete construction, Techno-Press, Vol. 4(1), pp 15-26. https://doi.org/10.12989/ACC.2016.4.1.015 GTR -Guide des Terrassements Routiers- (2000). Réalisation des remblais et des couches de forme (GTR), Guide technique, 102 p., fascicules I et II, références SETRA : D9233-1 et D9233-2), SETRA- LCPC, 2ème édition, juillet 2000.

GTS (2000). Traitement des sols à la chaux et/ou aux liants hydrauliques - Application à la réalisation des remblais et des couches de forme, Guide technique, 246 p., référence SETRA : D9924, SETRA-LCPC.

FERBER V., AURIOL J.-C., CUI Y. J., MAGNAN J.-P. (2009). On the swelling potential of compacted high plasticity clays. Engineering Geology, Vol. 104, pp 200-210. https://doi.org/10.1016/j.enggeo.2008.10.008

KOUASSI P. K., BREYSSE D., GIRARD H., POULAIN D. (2000). A new technique of kneading compaction in the laboratory, Geotechnical Testing Journal, ASTM, pp 72-82. https://doi.org/10.1520/GTJ11125J

LEVACHER D., JAIN S., PONTUS P., SEIFI S., HAQUIN S., HAQUIN S., TSHINKUNKU E. B. (2017). A method to compact dry mortars made with by-products, Geo-Environmental Engineering GEE 2017, May 19-20, Seoul National University, Seoul, 6 p.

MEKERTA B., SEMCHA A., RAHMANI F., TROALEN J.P. (2008). Erosion spécifique et caractérisation de la résistance au cisaillement des sédiments du barrage de Fergoug, $X^{\text {ème }}$ Journées Nationales Génie Côtier-Génie Civil, Sophia Antipolis, 14-16 octobre 2008, pp 135-144. https://doi.org/10.5150/jngcgc.2008.013-M

REMINI B., HALLOUCHE W. (2007). Studying sediment at Algeria's dams. International water power and dam construction 59, pp 42-45.

SEKLAOUI O. (2016). Valorisation des sédiments de barrage d'El Merdja Sidi Abed : étude techno-économique. Thèse de doctorat, Université Mouloud Mammeri de TiziOuzou, Algérie, $119 \mathrm{p}$.

TOUMI A., REMINI B (2020), Zardezas: a dam that is silting up? Larhyss Journal, Vol. 43, pp 181-196.

ZRI A. (2010). Mise en place d'une nouvelle approche de formulation d'une matrice cimentaire à base de sable de dragage : application aux bétons de sables et granulats. Thèse de doctorat, Université de Lille 1, 201 p. 